\title{
Application of a Touch-Probe Array for the Parallel Measurement of Microstructures
}

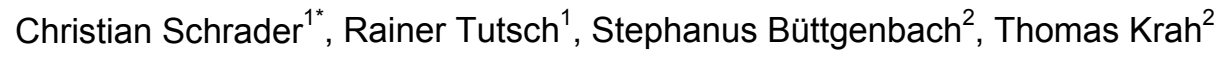 \\ ${ }^{1} \mathrm{TU}$ Braunschweig, Institute of Production Measurement Technology, \\ Schleinitzstrasse 20, 38106 Braunschweig, Germany, http://www.iprom.tu-bs.de/ \\ ${ }^{2}$ TU Braunschweig, Institute for Microtechnology, http://www.imt.tu-bs.de/ \\ Alte Salzdahlumer Straße 203, 38124 Braunschweig, Germany \\ *E-mail: c.schrader@tu-bs.de, Phone: +49 (0) 531 391-7039, Fax: +49 (0) 531 391-5837
}

\begin{abstract}
This paper deals with the development of a coordinate measuring machine for parallel tactile probing of microstructures. The particular challenges due to the parallel probing will be discussed and the concluding results regarding the construction, the control and the calibration will be outlined.
\end{abstract}

\section{Introduction}

Microstructures differ from microelectronic devices by a substantial higher aspect ratio and steep slopes. This is a limiting factor for optical measurement methods. For such large aspect ratios tactile measurement is a more promising approach. But for the production of microstructures tactile measurement methods currently only have little importance because they are adapted insufficiently to their specific needs. Microstructures are produced with batch processes with the characteristics that many elements are manufactured simultaneously on the same silicon wafer. This parallelism and the fact that in general there are multiple probing points needed to describe a single feature is in marked contrast to the sequential probing of conventional tactile measurement. This causes a high expenditure of time to measure all elements of a wafer. For this reason usually only spot checks are applied. Therefore it is the objective of the presented project to probe multiple elements at the same time to reduce the overall measurement time.

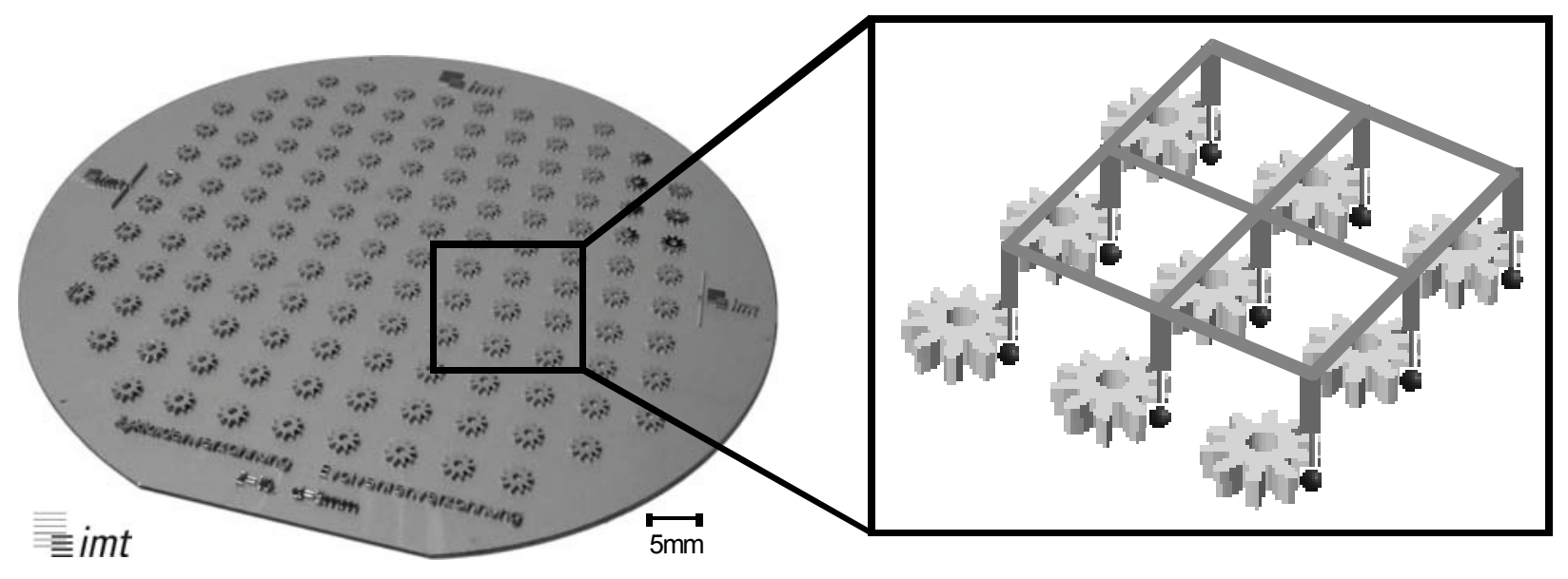

Fig. 1 Parallel probing at micro gear wheels on a wafer

\section{Approach}

The investigated approach to increase the measurement speed is to parallelize the measuring process by using multiple micro probes simultaneously. Fig. 1 shows the principle. The micro probes are arranged in an array with the same spacing like the objects on the wafer (gear wheels in Fig. 1). The probe array is moved in such a way that each probing ball gets in contact with the target point at the corresponding 
object. It is necessary to align the orientation of the array to the wafer before starting the real measurement in order to measure the same point on each surface. Following to this the array is moved to a position where every probe is in contact with the surface and the deflections of all touch probes have about the same magnitude. Because of the tolerances of the array and of course the objects on the wafer this will become a challenging part of the work. To facilitate the alignment of the array three rotational DOFs are needed.

\section{The Micro-CMM}

Key aspects of the activity are the parallelization of the probing, the calibration and the development of probing strategies, not to build a CMM with maximum precision usable for large wafers. The main design criteria therefore are:

- 6 degrees of freedom (DOF) between probe array and object

- good view to the array and object to use auxiliary cameras for interactive probing

- $\quad$ easy procedures to change the array and the object

Fig. 2 shows the CMM. Two goniometers and a rotation stage are attached to a cantilever mounted on top of a pillar and carry the touch probe array. The goniometers and the rotation stage are aligned in such a way that all three rotational axes intersect in one point which is in the plane of the probing balls of the array. This way the effective resolution of the stages gets maximized. The rotation angle of these stages is about $\pm 2.5^{\circ}-5^{\circ}$.

Below the array there is a 3D translational stage which holds the object to be measured. The maximum travel distance is about $15 \mathrm{~mm}$. After the alignment of the array this stage generates the movement for the measurement process. The split of the kinematic chains into a rotational and a translational part decreases the overall positioning error.

The main pillar consists of three steel plates in an $\mathrm{H}$-arrangement. This allows a good view from the backside. To change the array without damage it is very important to have a good access to its socket. Therefore the upper cantilever is mounted on a kinematic base which allows a statically determined positioning with high repeatability and makes it easy to separate the cantilever from the pillar.

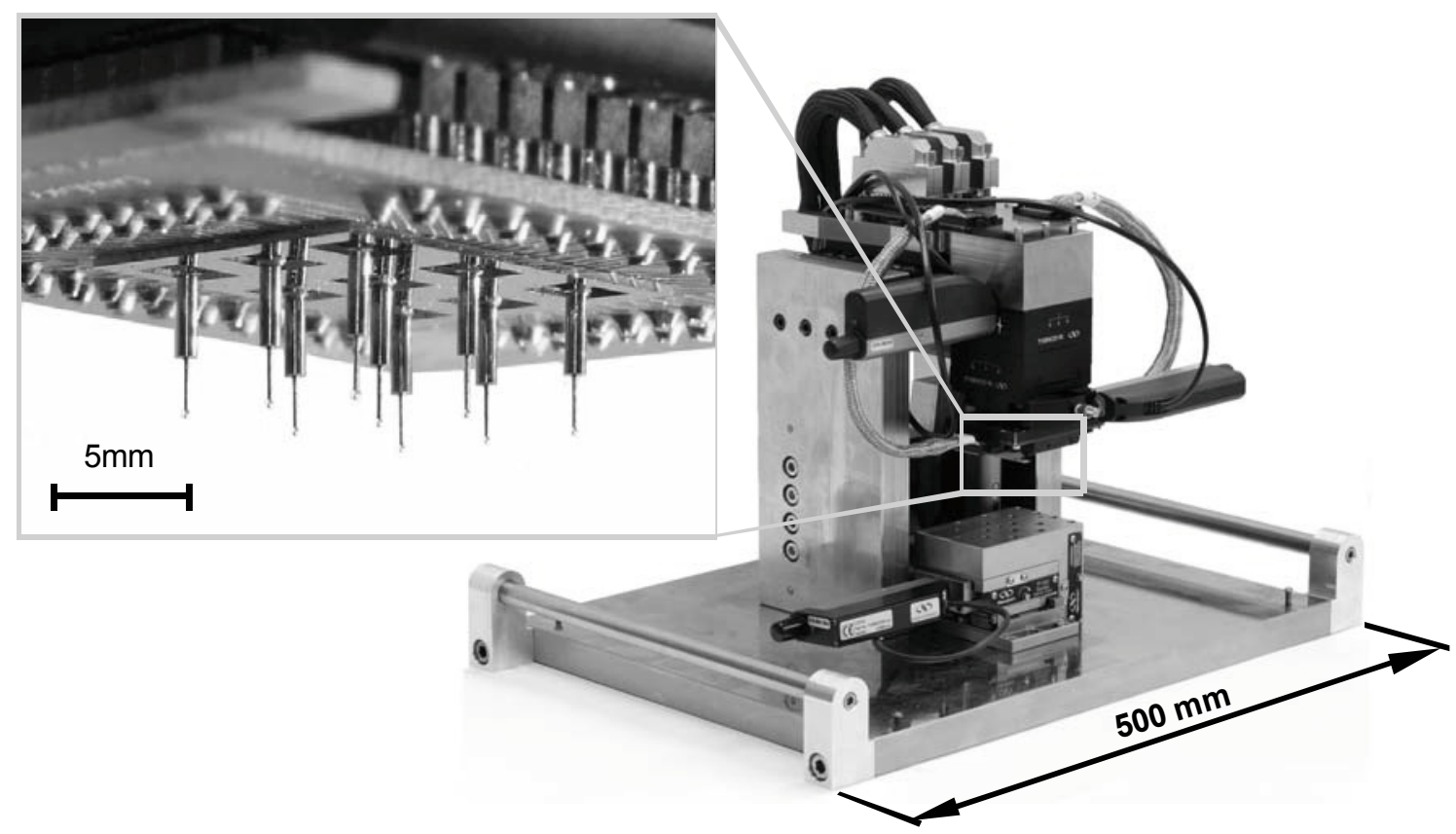

Fig. 2 Micro-CMM with probe array

The probe array itself is build of $3 \mathrm{D}$ micro touch probes which are manufactured from a single silicon wafer. Each probe consists of a membrane with a boss structure in the middle that holds the probing 
stylus with a ruby ball with $300 \mu \mathrm{m}$ diameter. A deflection of the probe leads to a deformation of the membrane which gets measured by piezo resistive sensors. The probes themselves are glued onto the boss structure. The whole array is mounted on a carrier PCB by using flip chip technique. With this board the array can be plugged into a ZIF socket to ensure a low strain of the array.

The stages are driven by DC motors and integrated gears. The minimum step size is $100 \mathrm{~nm}$ and the positioning error after calibration with a laser interferometer is below $0.3 \mu \mathrm{m}$ per actuator.

\section{Control of the CMM}

The structure of the control loop of the CMM is shown in Fig. 3. The probe array is connected to a set of instrumentation amplifiers. They amplify the sensor signals by a factor of 2000 . The signals get processed by the core component of the control, a computer with a realtime OS (Pharlap). It handles the time critical parts of the control like A/D conversion of the sensor signals, the computation of the resulting 3D probe deflection and the check for special events like probe-surface contact or maximum deflection which need a strict and immediate response. In most cases this means a stop of the actual movement. All these tasks are repetitively processed with a frequency of $100 \mathrm{~Hz}$.

The control of the actuators is done by a motion controller. It drives the actuators with jerk free velocity profiles which minimizes the vibration of the system. The controller is able to group the motors and let them drive a predefined trajectory. This mode will be used in a later stage of the project when it comes to scanning measurement of surfaces.

For the operation of the CMM there is another computer which features the user interface. This separation of functionality is needed to ensure a determinate timing behaviour of the realtime system. The operation computer provides elements for interactive movement of the CMM, script execution for writing complex measurement tasks and the storage of the measured data. The chosen scripting language is "Lua". Both computers are programmed using LabVIEW which features a realtime module and tools for distributed programming. The communication between the computers is achieved via Ethernet network.

One essential function of the software shall be explained. For a movement a set of deflection limits can be defined. This can be an arbitrary combination of the vector components of the deflection, the magnitude they have and the type of event, e.g. fall below a specific value or exceed it.

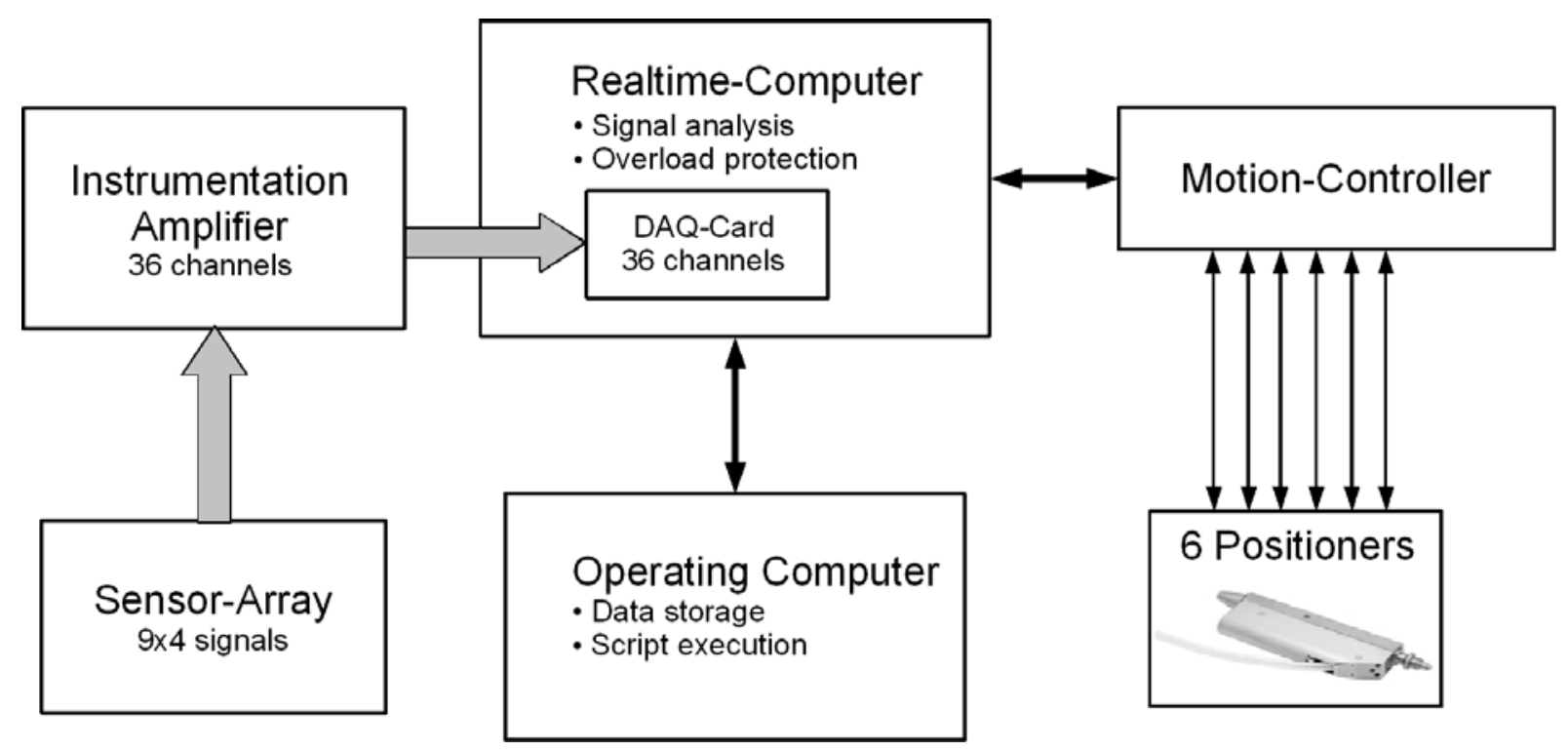

Fig. 3 Control structure of the CMM 
For example one can define:

stop when:

- for any probe the overall deflection (XYZ components) is above $10 \mu \mathrm{m}$

OR - for probe 1 or 2 the deflection in $X$ direction is larger than $2 \mu \mathrm{m}$

OR - for probe 3 the deflection is lower than $1 \mu \mathrm{m}$

This leads to some kind of event based programming. One has to define the set of limits and then just start a motion into the desired direction and wait until the motion stops. There is no need for manual limit checks inside the control scripts. These are done on the realtime system.

\section{Specific issues}

From this parallelization arise several challenges. First we have to consider that the positions of the probing balls are not ideal because of production tolerances. These differences superpose to those of the surface positions of each object. In order to measure simultaneously with each probe we have to push the array against the surface so that each probe gets in contact, the deflection limits $(20-100 \mu \mathrm{m})$ are not exceeded and the deflection is equalized for all probes. The last point is important to minimize the deformation of the array substrate and the resulting crosstalk between the probes and to equalize the contact force applied to the object.

The next issue is the compliance of the membranes which has to be determined as a function of direction in the calibration procedure. Because of the torque introduced by the weight of the stylus a small deflection occurs when the array is tilted. This needs to be respected when using nonlinear calibration curves because of the influence onto the operating point.

When probing microstructures the occurrence of adhesion effects is expected. This may inhibit the use of dynamic measurement with extrapolation to zero contact force as used with conventional CMM. The adhesion and friction forces may lead to an additional torque that bends the probing styli which only have relative low stiffness. This torque affects the measurement result.

\section{Calibration}
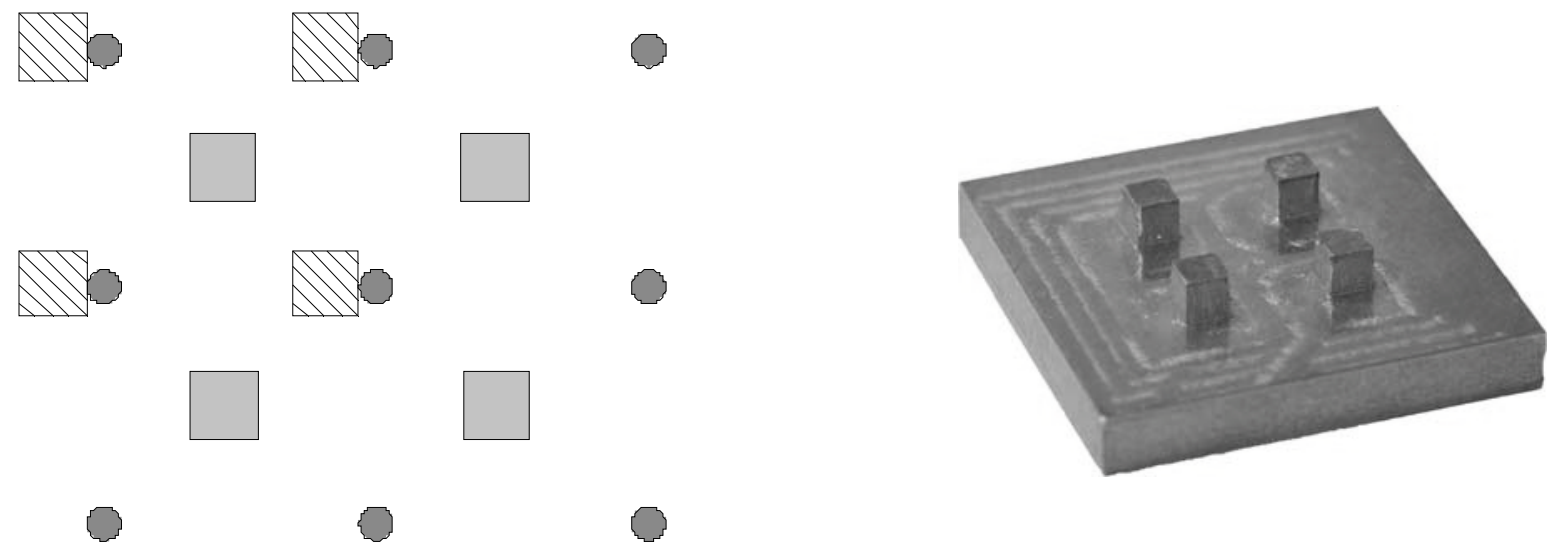

Fig. 4 Test object for first calibration step

Before a new touch probe array can be used a calibration procedure has to be applied. As mentioned above the constant checking of the probe deviation limits is an essential task of the realtime system. When inserting a new array the probe sensitivities are unknown so that no limit checks can be done. To determine these values manually by applying a specific deflection to a probe is a very time consuming task because this has to happen under visual control using cameras with large magnification. To automate this process the following approach (for a single probe) was chosen: The probe sensitivity is set to 1 , which means that we measure the output voltage of the sensor for $X, Y$ and $Z$ direction. In the first step the noise signal of the sensor without deflection is measured for some seconds. Then a contact limit 
of five times the standard deviation of the noise is defined. To move the probe by a known distance in every direction a simple small cuboid with an edge length of about $2 \mathrm{~mm}$ is used. It has only to get aligned roughly to hit the probe. Then the movement into one direction is started until the contact is detected. Then the movement stops. With the available arrays the probe got a deflection of about 1-2 $\mu \mathrm{m}$ then. Now we can do multiple well defined steps of some micrometers in the same direction and from the resulting voltage change the sensitivity for that direction can be calculated.

Fig. 4 shows an extended version of this calibration structure with four cuboids. This is needed to calibrate the whole array without realignment of the structure because of the limited range of the translational stage. Here some additional knowledge of the geometry is used to do some alignment of the orientation but the principle is the same like above.

\section{Acknowledgements}

The authors gratefully acknowledge funding of the project by Deutsche Forschungsgemeinschaft.

\section{References}

[Bue06] S. Büttgenbach, U. Brand, S. Bütefisch, Ch. Herbst, T. Krah, A. Phataralaoha, R. Tutsch: Taktile Sensoren für die Mikromesstechnik. VDI-Berichte, Nr. 1950, 2006, S. 109-118 